\title{
Le métier de communicant public et la démocratisation de l'action publique
}

Olivier Le Saëc

\section{(2) OpenEdition}

1 Journals

Édition électronique

URL : http://journals.openedition.org/communicationorganisation/3718

DOI : 10.4000/communicationorganisation.3718

ISSN : $1775-3546$

Éditeur

Presses universitaires de Bordeaux

\section{Édition imprimée}

Date de publication : 1 juin 2012

Pagination : 41-50

ISBN : 978-2-86781-821-9

ISSN : $1168-5549$

\section{Référence électronique}

Olivier Le Saëc, «Le métier de communicant public et la démocratisation de l'action publique », Communication et organisation [En ligne], 41 | 2012, mis en ligne le 01 juin 2015, consulté le 01 mai 2019. URL : http://journals.openedition.org/communicationorganisation/3718 ; DOI : 10.4000/ communicationorganisation.3718 


\title{
Le métier de communicant public et la démocratisation de l'action publique
}

\author{
Olivier Le Soëc ${ }^{1}$
}

Depuis les années 1980, la multiplication des organisations publiques dirigées par des acteurs politiques s'accompagne d'un développement du métier de communicant public. Alors que la communication politique est communément appréhendée comme une tentative de manipulation des citoyens ou comme une tentative de "viol des foules " au profit des intérêts du « Prince », en un peu plus de trente ans, le constat du développement d'un groupe d'acteurs professionnels de la communication publique, désormais omniprésents dans toute organisation publique, doit nous interpeller sur ce que révèle ce phénomène.

Face à cette situation, en s'inscrivant dans une approche compréhensive basée sur l'étude des chartes déontologiques des associations de communicants publics et sur des observations participantes lors de rencontres de professionnels, il apparaît nécessaire de s’interroger sur les caractéristiques du développement du métier de communicant public pour déterminer les mutations de l'action des organisations publiques. Nous prendrons conscience que le développement du métier de communicant public, généralement présenté "comme une réponse à un impératif de publicité (au sens kantien de diffusion d'information sur les questions d'intérêt général) et de proximité, voire de transparence, de la part des institutions publiques" ", est "une nécessité absolue qui traduit [des] mutations profondes ${ }^{3}$ ». Ainsi, nous réaliserons que le développement de ce métier répond à un processus de démocratisation de l'action publique à l'œuvre au sein des sociétés occidentales contemporaines car «le sinistre attribut des régimes totalitaires [s'est] ainsi transformé en un outil éminemment démocratique ${ }^{4} »$.

1 LERASS - CERIC, Université Montpellier 3 ; lesaecolivier@hotmail.com

2 OLLIVIER-YANIV C., La communication publique. Communication d'intérêt général et exercice du pouvoir, In Sciences de l'Information et de la communication. Objets, savoirs, discipline, Dir. OLIVESI S., Presses universitaires de Grenoble, 2006, p. 97.

3 WOLTON D, Sauver la communication, Champs Flammarion, Paris, 2005, p. 100.

4 OLLIVIER-YANIV C., L'État communiquant, Presses Universitaires de France, Paris, 2000, p. 89. 


\section{C\&O n41}

Ensuite, alors que «la communication publique [reste] très majoritairement à sens unique ", nous prendrons conscience que l'existence de chartes déontologiques pour les communicants publics met en exergue des questionnements de nature éthique sur leurs pratiques. Ainsi, au-delà de confirmer l'importance de "privilégier l'information et l'explication et ne pas céder aux seuls artifices de séduction ${ }^{6}$ ", l'affirmation d'une éthique professionnelle pour les communicants publics s'avère être une tentative de singularisation en établissant des "frontières " entre la communication politique et la communication publique en précisant que « le domaine de la communication publique se définit par la légitimité de l'intérêt général ${ }^{7}$ ".

\section{Le développement du métier de communicant public et la notion d'intérêt général}

Nous allons constater que le développement du métier de communicant public marque une évolution radicale de la conception de la communication des organisations publiques en s'inscrivant désormais dans les missions de service public tout en étant structuré autour de la notion d'intérêt général.

\section{Une évolution radicale de la conception de la communication publique}

La fin de la Seconde Guerre mondiale concrétisa une période intense de réflexions sur les pratiques des dispositifs d'information de l'État, suite à l'utilisation massive de la propagande par le régime de Vichy, qui contribua à faire émerger "l'idée selon laquelle le gouvernement est babilité à parler de sa gestion aux citoyens ${ }^{8} »$.

Ainsi, la période de l'après guerre fait émerger une conception radicalement différente de la communication des organisations publiques, appelée encore propagande ou information d'État, car «le sinistre attribut des régimes totalitaires [se transforme progressivement] en un outil éminemment démocratique ". Suite aux nombreuses réflexions sur les relations entre les gouvernés et les gouvernants de la démocratie représentative, il fut admis "le passage d'une conception de la propagande d'Etat comme l'apanage des régimes totalitaires à l'idée que l'information des citoyens par l'Etat peut être démocratique " car "la diffusion d'informations sur les actes et les décisions gouvernementales est un principe démocratiquement nécessaire ${ }^{11} »$.

5 BESSIÈRES D., «La définition de la communication publique : des enjeux disciplinaires aux changements de paradigmes organisationnels ", In Repenser la communication dans les organisations publiques, Communication E Organisation, $\mathrm{n}^{\circ} 35,2009$, p. 15.

6 ZÉMOR P., La communication publique, Presses universitaires de France, Paris, 2005, p. 23.

7 ZÉMOR P., op. cit., p 112.

8 OLLIVIER-YANIV C., L'État communiquant, op. cit., p. 86.

9 ibid., p. 89.

10 ibid., Paris, 2000, p. 62.

11 ibid., p. 95. 
Néanmoins, nous pouvons situer le développement du métier de communicant public plutôt dans les années 1970 à travers une conception managériale des relations entre l'administration et les administrés se basant sur le "principe selon lequel l'information représente un nouveau droit dans le contrat entre l'Etat et la nation ${ }^{12}$ ". Son développement sera renforcé suite à la promulgation des lois sur la décentralisation en 1982 qui conduisirent à la réorganisation administrative de l'État et au transfert de compétences aux collectivités territoriales et locales. Par la suite, au début du $21^{\text {ème }}$ siècle, les différentes lois sur la gouvernance et sur la démocratie de proximité confirmèrent ce changement de conception du rôle de l'État en matière de publicité et d'information des citoyens.

De plus, depuis une vingtaine d'années, comme pour les métiers de la communication en général, nous pouvons constater la constitution d'un réel champ professionnel autonome par le biais de la création de formations diplômantes en communication publique. Elles se sont développées et se sont organisées au sein des universités, des écoles spécialisées, des instituts d'études politiques et même au sein de l'École Nationale pour l'Administration confirmant ainsi une reconnaissance par le monde de l'enseignement supérieur et par la haute fonction publique. Même si, à défaut de l'existence d'un véritable concours « communication publique " pour intégrer la fonction publique en France, les communicants publics restent majoritairement des contractuels ou des fonctionnaires dont la formation initiale n'est pas la communication ${ }^{13}$, il demeure que la reconnaissance institutionnelle du métier de communicant public semble concrétiser le souhait de l'association "Cap'Com » que «les métiers de la communication publique [soient] exercés par des professionnels - quel que soit leur statut - formés et destinés à cette principale mission ${ }^{14}$ ».

D'autre part, l'étude des chartes déontologiques des associations de communicants publics fait apparaître que ces professionnels partagent des valeurs qui tranchent radicalement avec la communication politique pour des questions d'idéaux et de finalités morales. Pour ces professionnels, la communication publique "doit s'exercer hors de toute propagande ou falsification des faits et respecter la nécessaire transparence des informations dont elle dispose, tant à l'intention des décideurs que des usagers ${ }^{15}$ ". Ainsi, nous voyons que les chartes déontologiques des communicants publics peuvent apparaître comme une tentative d'établissement de frontières d'ordre éthique entre les professionnels de la communication publique et ceux de la communication politique.

12 OLLIVIER-YANIV C., La communication publique, op. cit., p. 99.

13 Association Communication publique. Le directeur de communication et internet dans les collectivités territoriales. Quelles influences sur le métier? Etude réalisée par Philippe Deracourt. Master Celsa en communication et information numériques. Janvier 2006.

14 Cap'Com. Charte déontologique de la communication publique. Association nationale des communicants publics. 2002.

15 ibid. 
Nous pouvons ainsi constater que le développement du métier de communicant public confirme une évolution radicale de la conception de la communication des organisations publiques, et donc, de la conception des relations entre les citoyens et les autorités publiques dans les sociétés démocratiques. Initialement conceptualisée comme une forme de propagande de l'État, désormais, la communication publique "s'inscrit dans le cadre des missions de l'information de service public et en respecte les règles en vigueur, tant déontologies que juridiques ${ }^{16}{ }^{\prime}$.

\section{La structuration du métier de communicant public autour de la notion de l'intérêt général}

Le développement du métier de communicant public fut renforcé par la structuration des acteurs professionnels du champ au sein de différentes associations à partir de la fin des années 1980. À ce jour, plusieurs associations regroupent les communicants des administrations, des collectivités territoriales et organisations ayant des missions de service public. Les associations des professionnels de la communication publique les plus connues se dénomment "Communication publique » et « Cap'Com».

L'association "Communication publique » fut créée en 1989 suite à différentes rencontres des professionnels de la communication institutionnelle publique, et, désormais, cette association œuvre au sein de la fédération européenne des associations de communication publique pour une reconnaissance professionnelle auprès des institutions européennes. En ce qui concerne plus particulièrement " Cap'Com ", lors de la première convention nationale des communicants publics en novembre 2002, il fut voté la charte déontologique de cette association, «la charte de Marseille ». Cette dernière met en avant que «devant le déploiement croissant de la société de l'information, la communication publique s'affirme aujourd'hui comme une nécessité incontournable des institutions et des états démocratiques ${ }^{17}$ ". Ainsi, pour cette association, la création de cette charte a pour but de "tracer les contours de l'exercice de la communication locale de service public, des droits et devoirs des professionnels qui l'exercent ${ }^{18}$ " et d' "élaborer "un contrat public" entre les partenaires qui la pratiquent : décideurs, employeurs et communicants ${ }^{19} »$. Clairement, ces chartes affirment les valeurs des professionnels de la communication publique car elles mettent en avant l'importance "d'éviter le mélange des genres entre communications institutionnelle et politique ${ }^{20}$ » et de "privilégier l'information et l'explication aux seuls artifices de séduction ${ }^{21}{ }^{\prime}$.

16 Cap'Com. Charte déontologique de la communication publique. Association nationale des communicants publics. 2002.

17 ibid.

18 ibid.

19 ibid.

20 ZÉMOR P., op. cit., p. 23.

21 ibid. p. 23. 
De plus, alors que les communicants publics se sont regroupés au sein de différentes structures associatives, la création de chartes déontologiques reflète des questionnements sur l'éthique et les valeurs de ces professionnels. Ces dernières précisent les principes d'action et les règles que doivent respecter les professionnels de ce champ. Ainsi, il apparaît que les membres de ces associations ont souhaité mettre en exergue les valeurs qu'ils partagent en déclarant que "le domaine de la communication publique se définit par la légitimité de l'intérêt général2 ».

Donc, alors que le développement du métier de communicant public traduit une évolution radicale de la conception des relations entre gouvernants et gouvernés, nous pouvons appréhender la création de chartes déontologiques pour les communicants publics comme la concrétisation d'une dynamique d'affirmation d'une valeur centrale et partagée par ces professionnels qui forge la reconnaissance de leur singularité, l'intérêt général.

\section{La tentative de démocratisation de l'action publique et l'utopie de la communication}

La mise en avant du développement du métier de communicant public autour de la notion d'intérêt général nous amène à appréhender ce phénomène comme une tentative de démocratisation de l'action publique qui est révélatrice de l'imprégnation de l'utopie de la communication au sein des sociétés démocratiques.

\section{La tentative de démocratisation de l'action publique}

Même si le développement des activités de communication des organisations et des autorités publiques peut être appréhendé comme la concrétisation de "l'Etat séducteur" ", nous savons que " malgré ces dérives, les métiers qui tournent autour de la communication traduisent un réel changement dans les rapports de travail, et sont révélateurs d'une mutation des rapports sociaux ${ }^{24}$ ».

Il apparaît que le développement du métier de communicant public traduit l'existence d'une dynamique de fond, traversant les sociétés démocratiques contemporaines, tentant de relégitimer l'État et les autorités publiques par le biais de la communication tout en confirmant un changement de conception des rapports existants entre les gouvernants et les gouvernés. Le développement du métier de communicant public symbolise une tentative d'adaptation des organisations publiques aux contraintes extérieures et à leur environnement social, tout en concrétisant la redéfinition des relations existantes. Alors, le développement du métier de communicant public doit être appréhendé comme une tentative de redéfinition des relations existantes entre l'Etat et les citoyens, car «l'argumentation [et la communication publique ont...] pour effet

22 ibid. p. 112

23 DEBRAY R., L'Etat séducteur, Gallimard, Paris, 1993.

24 WOLTON D., op. cit., p. 100. 
de tisser un lien réfléchi, d'établir une relation forte, de reconnaître implicitement l'existence d'une obligation; elle oblige un pouvoir à descendre de son piédestall

Plus précisément, les principes d'action et les règles contenus dans les chartes déontologiques des communicants publics tendent à préconiser l'adoption d'une conception positive des administrés « éclairés " par la communication. Ces derniers précisent que les communicants publics doivent "favoriser l'explication de façon à réduire les incompréhensions, les litiges ou les conflits et éviter le recours aux sanctions ${ }^{26}$ et qu'ils doivent considérer "ses destinataires comme des récepteurs actifs : des citoyens à part entière dont procède l'intérêt général, des hommes et des femmes rationnels et raisonnables, des personnes chacun et chacune en situation particulière $e^{27}$ ». Ainsi, «les messages ne sauraient être, dans leurs expressions, détournés à des fins particulières, [et] les communicateurs publics sont responsables à la fois devant l'autorité publique - l'organisation pour laquelle ils communiquent - et devant les citoyens $^{28}$ ". Autrement dit, le développement du métier de communicant public peut être appréhendé comme "une tentative à surmonter les effets du bouleversement des anciens rapports du visible et de l'invisible introduit par l'ordre démocratique dans le monde ${ }^{29}$ ".

Mais, bien que le métier de communicant public soit présenté comme se définissant par rapport à la légitimité de l'intérêt général, il demeure que les professionnels de la communication publique sont liés au pouvoir politique et leurs rapports au politique ne doivent pas être occultés. Il apparaît que le métier de communicant public reste dépendant du pouvoir politique et que ces professionnels exerçant dans les collectivités locales ont, en grande partie, comme responsable hiérarchique le chef ou les membres du cabinet de l'exécutif local dont ils dépendent. Clairement, ils demeurent fortement rattachés au pouvoir politique comme le changement des responsables de la communication peut le concrétiser à l'issue des échéances électorales. Ainsi, les communicants publics n'ont qu'une marge de manœuvre limitée pour respecter les valeurs éthiques contenues dans les chartes déontologiques car "la dépendance vis-à-vis du pouvoir politique reste manifeste ${ }^{30}$ ".

Donc, nous voyons que le développement du métier de communicant public, en tentant de s'autonomiser vis-à-vis du pouvoir politique par l'établissement de frontières d'ordre éthique entre la communication publique et la communication politique, répond à une dynamique de démocratisation à l'œuvre au sein des sociétés occidentales contemporaines, car, "l'ouverture donne au citoyen le droit de regard sur la cité et sur le monde. Des sanctuaires sacrés

25 ROSANVALLON P., La contre-démocratie. La politique à l'âge de défance, Éditions du Seuil, 2006, Paris, p. 310.

26 ZÉMOR P., op. cit., p. 23.

27 ZÉMOR P., op. cit., p. 22.

28 ibid.

29 ROSANVALLON P., op. cit., p. 316

30 PAILLART I., Les territoires de la communication, Presses Universitaires de Grenoble, Grenoble, 1993, p. 98. 
demeurent dans son esprit, mais son opinion lui est demandée sur ce qui a cessé d'être sacré, la conduite des affaires publiques, et la réflexion sur son destin lui est permise $e^{31} »$.

\section{La concrétisation de l'utopie de communication}

Les faits précédents peuvent nous permettent d'appréhender l'existence d'une corrélation entre le développement du métier de communicant public et la promotion de la communication comme une nouvelle utopie au sein des sociétés démocratiques.

En effet, en parallèle du phénomène de réorganisation de l'État et de transfert de compétences, le développement d'une réflexion sur la crise du lien social conduit à concevoir le métier de communicant public comme essentiel au lien social dans les sociétés démocratiques. Alors que les anciennes idéologies fédératrices des sociétés démocratiques sont en déliquescence, nous savons qu' " on ne parle jamais autant de communication que dans une société qui ne sait plus communiquer avec elle-même, dont la cohésion est contestée, dont les valeurs se délitent, que des symboles trop usés ne parviennent plus à unifier ${ }^{32}$ ". Il est ainsi désormais conceptualisé que le métier de communicant public "s'affirme comme un élément stratégique de la médiation entre les acteurs de la gouvernance et vise à créer, conforter ou renforcer le lien social entre les partenaires de l'action publique ${ }^{33} »$. Cette conception tend à conférer une importance accrue aux activités de communication dans les sujets traitant de la gouvernance et du lien social au sein des sociétés démocratiques, au point d'apparaittre comme faisant référence à l'illusion démocratique de "la suppression de tout système d'autorité ou de pouvoir »ou à "la croyance qu'il pourrait y avoir une représentation effectivement égale de tous dans la conduite de la chose publique ${ }^{34}$ ».

Nous voyons que le développement du métier de communicant public serait donc porteur de la promesse de l'idéal de la transparence conduisant à "requalifier négativement tout ce qui est de l'ordre du secret, du caché, du privé, de l'intime, de la profondeur, du non visible. L'annibilation concrète de ce «non visible» soit jugé «opaque» ne peut se faire alors qu'en s'attaquant aux barrières, aux frontières, à tout cloisonnement qu'empêchent la circulation de l'information, 'l'interconnexion généralisée' et la transparence finale du monde ${ }^{35} "$. Le développement du métier de communicant public serait "une nécessité absolue qui traduit [des] mutations profondes ${ }^{36}$ ", car il doit être appréhendé comme " une stricte réponse au déchirement du lien social, car, [la communication] a, en quelque sorte, absorbé une grande partie de l'espoir

31 MORIN E., La méthode, L'humanité de l'bumanité. L'identité humaine, Éditions du Seuil, Paris, p. 209.

32 SFEZ Lucien, La critique de la communication, Le Seuil, Paris, 1990, p. 5

33 Cap'Com. Charte déontologique de la communication publique.... op. cit.

34 MORIN E., Introduction pour une politique de l'homme, Éditions du Seuil, Paris, p. 106.

35 BRETON P., Le culte de l'Internet. Une menace pour le lien social ?, Éd. La Découverte et Syros, Paris, 2000.

36 WOLTON D., op. cit., p. 100. 
utopique dont nos sociétés sont capables ${ }^{37}$ ". Désormais, il est communément devenu admis que "le système politique qui assure la régulation de l'ordre social dépend principalement de la fonction de communication ${ }^{38}$ ", et que "l'extension de l'information a pour conséquence la participation de plus en plus grande du citoyen à la politique qui lui est appliquée $e^{39}$ ». Ainsi, cette utopie projective repose sur la conviction d'avoir trouvé la solution pour transformer la vie de la cité, car, le développement du métier de communicant public serait une étape pour la réalisation de "la société mondiale de l'information [pouvant] se définir comme un monde ainsi "transparent à lui-même», qui ferait enfin reculer les violences et constituerait l'idéal ultime de civilisation ${ }^{40} »$.

\section{Conclusion}

Force est de constater qu'en quelques décennies, il y a eu un changement radical de la conception de la communication publique au sein des sociétés démocratiques. Puisqu'" il n'y a pas de démocratie sans information et communication de masse ${ }^{41}$ ", le développement du métier de communicant public est la concrétisation d'un changement de conception des rapports existants entre les gouvernants et les gouvernés. C'est la confirmation de l'existence d'une tendance de fond de démocratisation des organisations publiques car, désormais, "l'information représente un nouveau droit dans le contrat entre l'Etat et la nation ${ }^{42}$ » obligeant " le pouvoir à descendre de son piédesta ${ }^{43}$ ". Ainsi, nous voyons que les évolutions dans le fonctionnement des sociétés démocratiques, en tant qu'organisations, se traduisent par une évolution des pratiques de communication des organisations publiques.

Au-delà d'être la marque de l'impératif de publicité des organisations publiques dans les sociétés démocratiques, le développement du métier de communicant public s'avère être la concrétisation d'une tentative de démocratisation de l'État, en tant que forme organisationnelle contraignante, tout en incarnant «l'utopie de la communication » comme «la Voix unique, qui seule peut unifier un univers ayant perdu en route tout autre référent ${ }^{44}$ ».

\section{BIBLIOGRAPHIE}

ASSOCIATION COMMUNICATION PUBLIQUE. Le directeur de communication et internet dans les collectivités territoriales. Quelles influences sur le métier?, Étude réalisée

37 BRETON P., L'utopie de la communication, l'émergence de «l'homme sans intérieur », op. cit., p. 69.

38 COTTERET J.-M., Gouverner, c'est paraître, Presses Universitaires de France, Paris, 1997. p. 113.

39 ibid.

40 BRETON Ph., L'utopie de la communication..., op. cit., p. 137.

41 WOLTON D., Naissance de l'Europe démocratique, Flammarion, Paris, 1993, p. 368.

42 OLLIVIER-YANIV C., La communication publique, op. cit. p. 99.

43 ROSANVALLON P., op. cit., p. 310.

44 SFEZ L., op. cit. 
par Philippe Deracourt, Master CELSA en communication et information numériques, Janvier 2006.

BESSIERES D., "La définition de la communication publique : des enjeux disciplinaires aux changements de paradigmes organisationnels », In, Repenser la communication des organisations publiques, Communication E Organisation, n 35, 2009.

BRETON P., L'utopie de la communication, l'émergence de "l'homme sans intérieur », La Découverte, 1992.

CAP'COM. Charte déontologique de la communication publique, Association nationale des communicants publics, 2002.

COTTERET J.-M., Gouverner, c'est paraître, Presses Universitaires de France, Paris, 1997.

DEBRAY R., L'État séducteur, Gallimard, Paris, 1993.

MORIN E., La méthode, L'humanité de l'bumanité. L'identité humaine, Éditions du Seuil, Paris.

OLLIVIER-YANIV C., La communication publique. Communication d'intérêt général et exercice du pouvoir, In OLIVESI S. (dir.), Sciences de l'Information et de la communication. Objets, savoirs, discipline, Presses universitaires de Grenoble, 2006.

OLLIVIER-YANIV C., L'État communiquant, Presses Universitaires de France, Paris, 2000.

PAILLART I., Les territoires de la communication, Presses Universitaires de Grenoble, Grenoble, 1993.

ROSANVALLON P., La contre-démocratie. La politique à l'âge de défiance, Éditions du Seuil, 2006, Paris.

SFEZ L., La critique de la communication, Le seuil, Paris, 1990.

WOLTON D., Sauver la communication, Flammarion, Paris, 2005.

WOLTON D., Naissance de l'Europe démocratique, Flammarion, Paris, 1993.

ZEMOR P., La communication publique, Presses universitaires de France, Paris, 2005.

Résumé : Le développement du métier de communicant public concrétise une évolution radicale de la conception de la communication publique dans les sociétés démocratiques. Désormais, rattaché à la notion d'intérêt général, le communicant public doit être défini comme exerçant une fonction de démocratisation et de régulation des tensions entre les autorités publiques et les citoyens, traduisant une nécessité absolue révélant des mutations profondes au sein des sociétés démocratiques contemporaines. Le développement du métier de communicant public nous amène à appréhender ce phénomène comme une tentative de démocratisation de l'action publique révélatrice de l'imprégnation de l'utopie de la communication au sein des sociétés démocratiques. Ainsi, les évolutions organisationnelles dans le fonctionnement des sociétés démocratiques se traduisent par une évolution des pratiques de communication des organisations publiques dans lesdites sociétés.

Mots-clés : Communicant public, Développement, Démocratisation, Utopie. 
Abstract : The development of public communication occupation reveal radical evolution of public communication within democratic societies. Consequently linked to the general interest, the public communication occupation must be conceptualized as exercising a function of democratization and regulation of tensions between the public authorities and the citizens, translating an absolute necessity revealing deep mutations inside contemporary democratic societies. The public communication occupation development could be approach as a try of democratization of public action revealing the impregnation of the utopia of communication within the democratic societies. So, evolutions in the functioning of the democratic societies, as organization, are translated by an evolution of the practices of communication of the public organizations in aforementioned societies.

Keywords : Public Communication occupation, Development, Democratization, Utopia 